МИНИСТЕРСТВО ОБРАЗОВАНИЯ И НАУКИ РОССИЙСКОЙ ФЕДЕРАЦИИ

ТОМСКИЙ ГОСУДАРСТВЕННЫЙ УНИВЕРСИТЕТ

\title{
МАТЕРИАЛЫ
}

VIII Международной молодежной научной конференции

«МАТЕМАТИЧЕСКОЕ

И ПРОГРАММНОЕ ОБЕСПЕЧЕНИЕ

ИНФОРМАЦИОННЫХ, ТЕХНИЧЕСКИХ

И ЭКОНОМИЧЕСКИХ СИСТЕМ»

Томск, 26-30 мая 2021 г.

Под общей редакиией И.С. Шмырина

Томск

Издательство Томского государственного университета 2021 


\title{
І. ИММЕРСИВНЫЕ ТЕХНОЛОГИИ, ТЕХНИЧЕСКОЕ ЗРЕНИЕ И ВИДЕОАНАЛИТИКА
}

DOI: $10.17223 / 978-5-907442-42-9-2021-1$

\section{РУКОВОДСТВО ПО САМОСТОЯТЕЛЬНОМУ ПРОЕКТИРОВАНИЮ И СБОРКЕ МАСШТАБНОЙ МОДЕЛИ С АВТОНОМНЫМ УПРАВЛЕНИЕМ НА ПРИМЕРЕ КОЕNIGSEGG ONE:1}

\author{
Дорожкин А.E. \\ Томский государственный университет \\ doliman100@gmail.com
}

Введение

Масштабная модель - это уменьшенная копия реального автомобиля. Бывают статические и радиоуправляемые модели. На данный момент можно купить либо готовую модель, либо готовый набор для самостоятельной сборки. Но что делать, если желаемая модель находится в недосягаемом ценовом диапазоне или не существует вовсе?

$\mathrm{C}$ появлением 3D-печати стало возможным производство подобных вещей в домашних условиях. Но на данный момент печать крупномасштабных моделей автомобилей слабо распространена, и найти какую-либо информацию по этому направлению очень сложно. Мы хотим предложить решение, которое поможет любому желающему, не обладающему навыками 3D моделирования, самостоятельно спроектировать и собрать свою масштабную модель. В данном руководстве изложены базовые техники и принципы, которые позволят достичь желаемого результата. Также здесь рассмотрены разные варианты достижения поставленных целей, описаны их преимущества и недостатки.

\section{1. Постановка задачи}

Предлагается реализовать модель со следующими свойствами в масштабе 1:8:

1. Сходство с оригиналом.

2. Большой уровень детализации.

3. Интерактивность - наличие подвижных частей, например, возможность открыть дверь или крышку моторного отсека.

4. Автономное управление.

В автомобиле можно выделить несколько ключевых элементов: кузов, подвеска и электронная схема. Всё это проектируется самостоятельно.

Немаловажным является выбор прототипа. Т.к. создание масштабной модели - это долгий процесс, то для рассмотрения был выбран автомобиль, на который будет не жалко затраченного времени. У Koenigsegg One:1 имеются уникальные дверные петли [1], и некоторое время ему принадлежал рекорд 0-300-0 км/ч среди серийный автомобилей. Кроме того, всего было выпущено шесть таких автомобилей, но изготовлено их было семь. В руководстве рассматривается седьмой автомобиль, который принадлежал компании-производителю, и использовался для экспериментов.

\section{2. Кузов}

Кузов является самой главной частью автомобиля, т.к. именно он отражает его облик. Отсюда возникает необходимость сделать его не отличимым от настоящего автомобиля. 


\section{1. Исходные данные}

Широко распространён метод самостоятельного моделирования кузова по проекциям. Данный метод требует наличия выдающихся способностей в области 3Dмоделирования. Кроме того, происхождение таких проекций зачастую неизвестно. Все находящиеся в открытом доступе проекции описанного здесь автомобиля основаны на проекциях, созданных дизайнером MillergoCG.

Самый достоверный способ передать облик настоящего автомобиля - это взять готовую 3D-модель. Для этого требуется только наличие достаточного количества времени на исправление модели. Никакого навыка 3D-моделирования не понадобится, т.к. в видимую геометрию кузова нельзя вносить никакие изменения.

Самый достоверный и полный источник моделей автомобилей - это видеоигры. Крупные разработчики заключают контракты с производителями автомобилей для включения эксклюзивных автомобилей в игру. Производители заинтересованы в том, чтобы их автомобили выглядели как можно более достоверно, поэтому они предоставляют реальные автомобили для точного 3D-сканирования [2].

Для подведения итога по двум этим методам нужно сказать, что проекции Millergo сняты с модели, которую он изготовил по заказу Electronic Arts для игры Need for Speed: Rivals.

Т.к. технология создания моделей для игр показана на примере видеоигры Forza Horizon 4, данное руководство основывается на модели из этой игры (рис. 1).

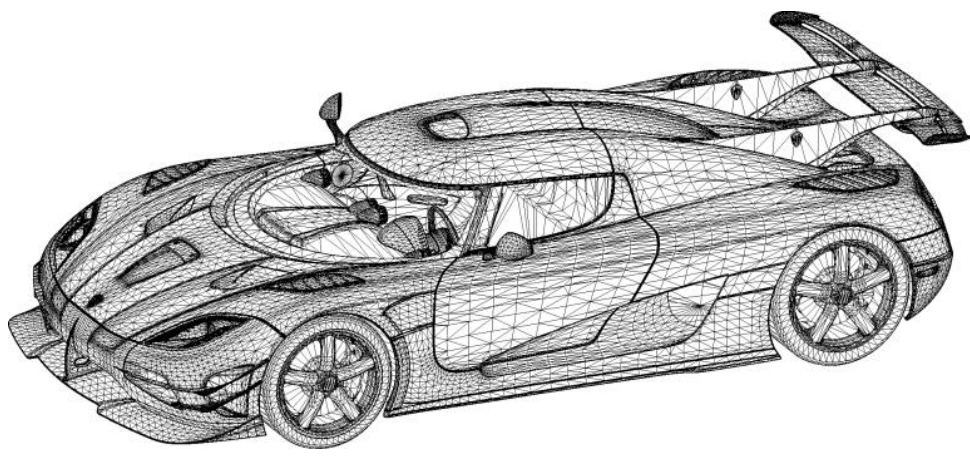

Рис. 1. Модель кузова Koenigsegg One:1

Модели из игр обычно извлекаются из дампов памяти видеокарты, сохранённых во время игры. Для извлечения моделей из серии игр Forza существует специальная программа 3DSimED.

\section{2. Обработка модели}

Извлечённая модель представляет из себя полигональную сетку. Для работы с такими моделями используется программа $3 \mathrm{ds}$ Мах. Очень важно при обработке модели не затрагивать внешнюю геометрию кузова. Обработка заключается в вырезании отдельных деталей из сетки, объединении разбитых на разные части деталей в одну, произведение булевых операций над деталями с пересекающимися поверхностями и придание оболочке кузова толщины. В конечном счёте, получается набор панелей, подобных тем, из которых собираются полномасштабные реплики из стекловолокна.

\section{3. Параметрическая модель}

Моделирование внутренних деталей происходит в программе параметрического моделирования Fusion 360. К таким деталям относятся: дверные петли, подвеска и различные крепления. Кузова реальных автомобилей также параметрические, но такие модели являются коммерческой тайной. Т.к. Fusion 360 плохо справляется с полигональными сетками, лицевая и внутренняя части объединяются в программе $3 \mathrm{ds}$ Max. 


\section{4. Изготовление}

Для полноразмерных реплик обычно используется трудоёмкая технология изготовления кузовов из стекловолокна. Преимущество данного метода заключается в том, что он не требует дорогостоящего оборудования.

При создании масштабной модели есть возможность распечатать готовую модель на 3D-принтере из пластика.

Распечатывание лучше начать с какой-то небольшой и несложной детали, чтобы отладить процесс, а на подготовку какой-то большой детали может уйти много времени. Например, можно начать с заготовки диска для колеса. Печать происходит в несколько этапов, самые первые модели нужны просто для понимания происходящего, они не должны обладать совершенной формой или продуманными креплениями, т.к. не будут использоваться в финальной сборке.

\section{3. Подвеска}

Конструкция подвески у гоночных радиоуправляемых моделей, доступных на рынке, схожа. Она направлена исключительно на повышение ходовых характеристик модели. С другой стороны, сделать функциональную копию подвески реального автомобиля в масштабе невозможно, в виду наличия очень мелких деталей.

Таким образом, для того, чтобы подвеска внешне напоминала свой прототип, но при этом обладала ходовыми характеристиками, не уступающими самым дешёвым моделям на рынке, при проектировании нужно принять во внимание обе конструкции.

\section{1. Исходные данные}

На сайте производителя [3] есть некоторое количество измеримых характеристик, таких, как колёсная база, дорожный просвет, радиус поворота и размеры колёс. Модель кузова выступает в качестве отправной точки, для того, чтобы детали подвески не пересекались с кузовными панелями.

В качестве источника информации могут выступать работы студентов, проходивших практику на заводе изготовителя, т.к. компания предоставляет им доступ к техническим моделям. В работе [4] одного из таких студентов мы нашли проекции задней подвески. Стоит отметить, что они совпадают, и даже дополняют модель из Need for Speed: Rivals, что говорит о высоком уровне достоверности обоих источников.

Некоторые детали изготавливать самостоятельно слишком сложно и нецелесообразно, например, амортизаторы и шаровые соединения. Вместо этого приобретаются стандартные заводские запасные части. Таким образом, их размеры тоже являются исходной информацией.

Некоторые характеристики можно косвенно вычислить из имеющихся. Так, угол поворота колёс находится из радиуса поворота, с учётом правила Аккермана.

\section{2. Проектирование}

Базовый принцип, который лежит в проектировании функциональной подвески, заключается в том, что все размеры должны быть обоснованными. Для примера возьмём проектирование задних рычагов, т.к. о них имеется достаточно много информации.

Сначала создаётся модель колёсного диска. Далее создаётся примерная модель задней подвески. После этого происходит подстройка деталей по ситуации. В данном случае в задней подвеске есть две детали, от которых можно оттолкнуться. В корпусе дифференциала заготовлены крепления под амортизаторы, длина и ход которых известны. При подборе длины рычагов следует руководствоваться принципом "чем больше - тем лучше".

Нижний рычаг ни от чего не зависит, поэтому он должен быть на столько длинным, на сколько это возможно. Верхний рычаг связан с амортизатором, т.е. нужно учитывать длину плеч, возникающих со стороны амортизатор, и со стороны колеса. Если 
плечо амортизатора будет короче плеча колеса, то на него будет воздействовать повышенное усилие, что приведёт к его низкой эффективности.

Нахождение оптимальной формы рычагов происходит путём использования тестовых сборок. Их суть заключается в создании неполной сборки модели, содержащей только исследуемые части. В данном случае сборка содержит только подрамник, рычаги, амортизатор и обод колеса. Сборка дублируется таким образом, чтобы в одном случае амортизатор находился в полностью сжатом, а в другом случае - в полностью разжатом состоянии, т.к. он определяет ход колеса. Если в одном из крайних положений поверхность рычага пересекается с поверхностью обода, необходимо изменить значения динамических параметров. Параметрами может быть как длина рычагов, так и их точка крепления к поворотному кулаку.

\section{3. Изготовление}

Для изготовления модель преобразуется в чертежи. В программе Fusion 360 для этого предусмотрен удобный функционал.

Для изготовления деталей подвески 3D-печать не подходит, т.к. на подвеску приходятся большие усилия. При изготовлении из пластика все узлы должны были бы быть очень толстыми, или даже монолитными [5], что существенно влияет на внешний вид. Поэтому подвеска изготавливается преимущественно из листового металла.

Сначала сгибается набор маленьких металлических деталей. Затем, путём использования оправок, сваривается в такие пространственные конструкции, как передний подрамник. Отверстия в детали сверлятся на координатном столе после того, как деталь сварена, для обеспечения соосности отверстий.

Отдельно стоит выделить процесс изготовления колёс, т.к. они одновременно являются и частью силовой конструкции, и важным элементом внешнего вида. Необходимость в изготовлении колёс возникает ввиду того, что колёса для радиоуправляемых моделей стандартизированы, и их существует всего несколько разновидностей. Колесо состоит из двух частей - диск и покрышка.

Диск изготавливается из двух частей. Силовая часть вытачивается из металла, а сверху накладывается накладка, распечатанная на принтере. Таким образом, достигается высокая прочность с сохранением внешнего вида.

Покрышки изготавливаются путём отливки из полиуретана с использованием силиконовой формы. Данная методика широко распространена среди владельцев гоночных RC-моделей ввиду дороговизны заводских комплектов покрышек. В данном случае силиконовая матрица снимается не с настоящей покрышки, а с твёрдой заготовки из пластика, распечатанной на 3D-принтере.

\section{4. Электронная схема}

Модель оснащается большим количеством иллюминации и сервоприводами, приводящими в движение такие части, как заднее антикрыло. Кроме того, сама модель приводится в движение мощным электромотором и имеет на борту видео камеру. Bсе эти компоненты требуется как-то контролировать и подавать на них требуемое напряжение.

Для повышения компактности данного комплекса предлагается разработать свою плату расширения, на которой будут расположены все необходимые управляющие компоненты.

Т.к. задача обработки изображения требует вычислительной мощности, недоступной на микроконтроллерах, предлагается использовать для этой цели одноплатный компьютер Raspberry Pi 4 Model B. Таким образом, получается конструкция, занимающая мало места, но обладающая большим потенциалом. 


\section{5. Автономное управление}

Заключительная часть работы над масштабной моделью - это написание программы управления. Рассмотрим вариант с автономным управлением. Т.к. автономное управление предполагает решение какой-то задачи в автономном режиме, сначала нужно поставить эту задачу. Для примера берётся задача, поставленная в рамках фестиваля по образовательной робототехнике «Hello, Robot»[6]. Задача заключается в том, что роботу необходимо двигаться вдоль кривой, нанесённой на белое поле краской, не отражающей свет.

В качестве входных данных используется только изображение с камеры, закреплённой в передней части модели. Для взаимодействия с изображением используется библиотека OpenCV.

Алгоритм обработки состоит из нескольких этапов.

Первым делом нужно найти на изображении саму линию. Для этого анализируется полоса пикселей, находящаяся в нижней части изображения. Анализ заключается в преобразовании цветов пикселей в градации серого. Для устранения шумов в виде плавных переходов между белым и чёрным цветом, например, лёгких теней, используется фильтр, находящий разницу между соседними пикселями. Таким образом, в местах резкого перехода между цветами получаются всплески, а там, где цвета перетекают плавно, всплески (даже если и есть) - небольшие. В качестве отправной точки берётся точка, находящаяся между самым большим положительным и отрицательным всплесками.

Преимущество камеры перед датчиками цвета, закреплёнными под передним бампером, заключается в прогнозировании движения [7], поэтому после нахождения отправной точки аналогичным образом анализируются пиксели, находящиеся на заданном расстоянии от неё, т.е. теперь анализируется не прямая, а окружность. Найденная на данном шаге точка является продолжением линии, найденной на предыдущем шаге.

Если повторить предыдущий шаг, получим набор точек, отражающий положение линии во всём поле обзора камеры. Таким образом, можно выполнить прогнозирование движения и заранее подготовиться к вхождению в поворот.

Данные шаги позволяют справиться с поставленной задачей. Теперь можно её расширить, запустив модель на «восьмёрке» - это тип замкнутой трассы с перекрёстком в центре. Таким образом, представленного алгоритма оказывается недостаточно, т.к. появляется необходимость различать пути, образующие перекрёсток, и выбирать тот, что приведёт к наименьшему отклонению от текущего курса.

Для решения данной проблемы, когда в процессе анализа окружности возникает ситуация, при которой её диаметра оказывается недостаточно, она расширяется, т.е. теперь анализируется значительно большая область, в которую помещается сразу весь перекрёсток.

\section{Заключение}

В качестве доказательства эффективности автономной программы управления можно сказать, что по результатам соревнования, из которого было взято задание, данная модель заняла первое место в старшей категории гонок по линии. Также данная модель принимала участие во всероссийском этапе чемпионата RoboCup Russia Open 2017, который проходил в рамках форума молодых учёных U-NOVUS, по итогам которого в рамках дисциплины Rescue Line заняла шестое место.

В рамках данного проекта было построено две модели. Первая модель использовалась для тестирования алгоритма автономного управления, она и принимала участие в соревнованиях. Вторая модель разрабатывается в соответствии с изложенными здесь техниками. На данный момент изготовлена действующая подвеска из металла, на которую установлены заводские колёса. 


\title{
ЛИТЕРАТУРА
}

1. Koenigsegg C. Door hinge. [Электронный pecypc] // Google Patents. URL: https://patents.google.com/patent/US10662687B2/en (дата обращения: 11.06.2021).

2. The Making of Forza Motorsport 4: Car Sourcing [Электронный ресурс] // Официальный ҮоuТube канал Turn 10 Studios. URL: https://www.youtube.com/watch?v=CWb7cCS16TM (дата обращения: 11.06.2021).

3. Характеристики Koenigsegg One:1 [Электронный ресурс] // Официальный сайт Koenigsegg. URL: https://www.koenigsegg.com/car/one1/ (дата обращения: 11.06.2021).

4. Oliva M. Rear Cross Frame Re-Design and Optimization in Carbon Fiber. [Электронный ресурс] // База знаний Политехнического университета Каталонии. URL: https://upcommons.upc.edu/handle/2099.1/24276 (дата обращения: 11.06.2021).

5. Sköld O. The 3D-printed Koenigsegg Agera RS [Электронный pecypc] // The blog of Olle Sköld. URL: http://depronized.com/2019/04/29/the-3d-printed-koenigsegg-agera-rs/ (дата обращения: 11.06.2021).

6. Положение о проведении Фестиваля "Hello, Robot". [Электронный ресурс] // Официальный сайт центра "Планирование карьеры". URL: http://cpc.tomsk.ru/priglashaem-k-uchastiyu-v-festivale-po-obrazovatelnoyrobototehnike-hello-robot/ (дата обращения: 11.06.2021).

7. Дорожкин А.Е., Дорожкин Е.П., Изюмов А.А. Использование набора Lego Mindstorms 9797 для решения задачи "цветная сортировка" // Научная сессия ТУСУР-2017 : материалы Международной научно-технической конференции студентов, аспирантов и молодых ученых, посвященной 55-летию ТУСУРа. Томск, 10-12 мая 2017 г. - В-Спектр, 2017. - Ч. 1. - С. 263-266.

DOI: $10.17223 / 978-5-907442-42-9-2021-2$

\section{ДЕТЕКТИРОВАНИЕ СТАНДАРТНЫХ ДОРОЖНЫХ ЗНАКОВ НА ИЗОБРАЖЕНИЯХ}

\author{
Рудов В.А., Приступа А.В., Скворцов А.В. \\ Томский государственный университет \\ rudov.vlad2013@gmail.com, andreypr@mail.ru
}

\section{Введение}

Задача распознавания дорожных знаков на фото- и видеоизображениях достаточно часто возникает в самых разных отраслях: в дорожном и железнодорожном хозяйстве при паспортизации и оценке уровня содержания дорог, в беспилотных автомобилях для коррекции цифровой модели дороги при движении по заданному навигационному маршруту [1].

После принятия в 1968 г. Венской конвенции о дорожном движении основная масса знаков в мире была стандартизирована [1], и только небольшое число знаков допускалось оформлять нестандартно текстовыми надписями и стрелками. Такие знаки в России называются знаками индивидуального проектирования (ЗИП) [2]. И если распознавание таких знаков ещё алгоритмически возможно, то их автоматизированная интерпретация весьма сомнительна. Именно поэтому в основном решается задача распознавания стандартных знаков.

Существующие методы решения задачи детектирования объектов на изображении можно разделить на 3 категории [3]:

- опирающиеся на цветовые признаки;

- опирающиеся на форму и контур;

- на основе нейронных сетей.

За последние годы методы, опирающиеся на цветовые признаки, форму и контур объектов, в реальных условиях показали свою недостаточно высокую эффективность, Т.К.:

- объект на изображении может находиться не под прямым углом или может оказаться повернут вокруг своей оси;

- цвета на изображении могут быть искажены ввиду различных погодных условий или освещения;

- знак может частично перекрываться другим объектом. 